\title{
MET Y1253D-activating point mutation and development of distant metastasis in advanced head and neck cancers
}

\author{
Pirus Ghadjar · Wieslawa Blank-Liss • \\ Mathew Simcock · Ivan Hegyi · Karl T. Beer • \\ Holger Moch · Daniel M. Aebersold · Yitzhak Zimmer
}

Received: 26 January 2009/ Accepted: 26 June 2009/Published online: 29 July 2009

(C) Springer Science+Business Media B.V. 2009

\begin{abstract}
We investigated if the MET-activating point mutation Y1253D influences clinical outcomes in patients with advanced squamous cell carcinoma of the head and neck (HNSCC). The study population consisted of 152 HNSCC patients treated by hyperfractionated radiotherapy alone or concomitant with chemotherapy between September 1994 and July 2000. Tumors were screened for the presence of the MET-activating point mutation Y1253D. Seventy-eight patients $(51 \%)$ received radiotherapy alone, 74 patients (49\%) underwent radiotherapy concomitant with chemotherapy. Median patient age was 54 years and median follow-up was 5.5 years. Distant metastasis-free survival, local relapse-free survival and overall survival were compared with MET Y1253D status. During follow-up, 29 (19\%) patients developed distant metastasis. MET Y1253D was detected in tumors of 21 out of 152 patients (14\%).
\end{abstract}

P. Ghadjar · W. Blank-Liss · D. M. Aebersold · Y. Zimmer Department of Radiation Oncology, Inselspital, Bern University Hospital and University of Bern, Freiburgstrasse, 3010 Bern, Switzerland

W. Blank-Liss · Y. Zimmer $(\bowtie)$

Department of Clinical Research, University of Bern, Murtenstrasse 35/E-807, 3010 Bern, Switzerland

e-mail: yitzhak.zimmer@insel.ch

M. Simcock

Swiss Group for Clinical Cancer Research Coordinating Center

(SAKK CC), Effingerstrasse 40, 3008 Bern, Switzerland

I. Hegyi · H. Moch

Department Pathology, University Hospital Zürich,

Schmelzbergstrasse 12, 8091 Zürich, Switzerland

K. T. Beer

Radiation Oncology Centre Biel, Rebenweg 38, 2501 Biel,

Switzerland
Distant metastasis-free survival $(P=0.008)$ was associated with MET Y1253D. In a multivariate Cox regression model, adjusted for T-category, only presence of MET Y1253D was associated with decreased distant metastasisfree survival: hazard ratio $=2.5$ (95\% confidence interval: 1.1, 5.8). The observed association between MET Y1253Dactivating point mutation and decreased distant metastasisfree survival in advanced HNSCC suggests that MET may be a potential target for specific treatment interventions.

Keywords MET $\cdot$ Mutation $\cdot$ HNSCC $\cdot$ Metastasis

\begin{tabular}{|c|c|}
\hline \multicolumn{2}{|c|}{ Abbreviations } \\
\hline SAKK CC & $\begin{array}{l}\text { Swiss Group for Clinical Cancer Research } \\
\text { Coordinating Center }\end{array}$ \\
\hline HNSCC & $\begin{array}{l}\text { Squamous cell carcinoma of the head and } \\
\text { neck }\end{array}$ \\
\hline EGFR & Epidermal growth factor receptor \\
\hline $\mathrm{HGF} / \mathrm{SF}$ & Hepatocyte growth factor/scatter factor \\
\hline DMFS & Distant metastasis-free survival \\
\hline LRFS & Local relapse-free survival \\
\hline OS & Overall survival \\
\hline $\mathrm{RCT}$ & Radiochemotherapy \\
\hline RT & Radiotherapy \\
\hline PCR & Polymerase chain reaction \\
\hline PNA & Peptide nucleic acid \\
\hline SAS & Statistical analysis systems \\
\hline CI & Confidence interval \\
\hline HR & Hazard ratio \\
\hline
\end{tabular}

\section{Introduction}

Development of distant metastasis has a crucial impact on therapy and prognosis of advanced squamous cell 
carcinomas of the head and neck (HNSCC). If metastatic disease occurs, overall survival is significantly decreased [1]. During the last decade the incidence of local relapse [2] and distant metastasis [3, 4] was significantly reduced by the establishment of either postoperative or definitive concomitant radiochemotherapy, leading to an increase in overall survival [4-8]. However, the development of local and/or systemic tumor relapse is still considerable and remains to be further improved. An increasing body of evidence points towards the impact of various growth factor signaling pathways in the oncogenesis and prognosis of HNSCC, representing attractive anti-neoplastic targets [9-11]. Compelling evidence from both experimental and translational studies on the impact of the epidermal growth factor receptor (EGFR) signalling in prognosis and treatment failure of HNSCC led recently to the successful introduction of cetuximab, a monoclonal anti-EGFR antibody [12]. MET, the tyrosine kinase receptor for hepatocyte growth factor/scatter factor (HGF/SF) is involved in carcinogenesis, tumor progression and promotes aggressive cellular invasiveness which is strongly associated with tumor metastasis [13-15]. MET is not only involved in onset but also in maintenance of experimental metastasis [16]. Due to its extensive involvement in cancer development and its expression in various human cancers [17], MET has been identified as an attractive anti-cancer target against which numerous inhibitors have been developed, some of them being currently tested in clinical trials. Aberrant MET signaling can either be achieved by autocrine or paracrine HGF/ SF signaling, gene overexpression or point mutations leading to constitutive kinase activity, or by gene amplification or rearrangement. The detection of point mutations is challenged by the fact that clinical specimens often contain only a small number of tumor cells within a large background of normal tissue and that somatic point mutations are sometimes present only in a subset of the tumor cells. However, the detection of low-abundance mutations is important since clonal expansion of these cells can lead to a more aggressive tumor phenotype and patients with $M E T$ mutations might differentially benefit from $M E T$ targeted therapy [18]. The MET Y1253D activating point mutation was first identified in HNSCC [19] and we have later described its prevalence and clinical impact in a retrospective cohort of radiotherapy-treated patients with oropharyngeal cancer [20]. MET Y1253D has also been shown to couple and activate signalling pathways, which confer a motile-invasive phenotype on cancer cells [21]. As a next step, the current study was conducted to determine in a prospective setting if the MET Y1253D mutation is associated with distant metastasis-free survival (DMFS), local relapse-free survival (LRFS) or overall survival (OS) in advanced HNSCC.

\section{Materials and methods}

Patients and clinical protocol

Pre-therapy tumor samples were analyzed from patients who were treated in a multicenter phase III trial (SAKK 10/94) investigating the use of two courses of cisplatin simultaneously with hyperfractionated radiotherapy (RCT) compared to radiotherapy (RT) alone [22]. The protocol for SAKK 10/94 was approved by local ethics review boards and all patients gave informed consent. The trial was registered at the National Institute of Health (www.clinicaltrial.gov; identifier number: NCT00002654).

Tissue from 152 of the 224 patients enrolled in the trial were available for analysis. Tumor material for the remaining 72 patients could not be collected from the pathology departments of the participating centres. Inclusion and exclusion criteria of the trial have been previously described [22]. Briefly, all eligible patients had invasive squamous cell carcinoma of the oral cavity, oro- or hypopharynx, or larynx at different clinical stages. Those with primary tumor sites in the nasopharynx or paranasal sinuses were excluded from the trial. Tumors were classified according to the tumor-node-metastasis staging system (International Union Against Cancer, edition 4, 1987). Each eligible participant underwent clinical evaluation by a multidisciplinary team. This evaluation included a chest radiograph, computed tomography of the head and neck, and examination of the upper aerodigestive tract under general anesthesia. The judgement of operability was left to the treating surgeon. To identify and exclude patients with distant metastases, liver ultrasound or abdominal computed tomography and bone scintigraphy were mandatory for all patients with hypopharynx cancer and/or positive lower neck nodes. With the exception of those having basal cell carcinoma of the skin, squamous cell carcinoma of the lip, or in situ carcinoma of the uterine cervix, all patients with prior malignancies were excluded from the trial. All patients were seen by head and neck specialists weekly if $\geq$ grade 3 mucositis occurred, and otherwise at 6 weeks after completion of trial therapy, every 2 months during the first year, every 3 months during the second year and every 6 months thereafter until death.

\section{Radiotherapy and chemotherapy}

Delivery of treatment has been described previously [22]. All 152 patients analyzed were treated with radiotherapy up to a median total dose of 74.4 Gy (72-76.8 Gy), during 7 weeks, with 1.2 Gy given twice daily with an interfraction interval of $6 \mathrm{~h}$. 
Cisplatin $20 \mathrm{mg} / \mathrm{m}^{2}$ was administered with intravenous hydration on 5 consecutive days during weeks 1 and 5 or 6 of radiotherapy, $1.5 \mathrm{~h}$ before the afternoon radiotherapy session. A total of 71/74 (96\%) patients received at least 1 cycle of cisplatin and 55/74 (74\%) patients received a total of 2 cycles.

\section{DNA extraction and genotyping}

All the pre-therapeutic formalin fixed biopsies were reviewed by the local pathologist to assure the presence of squamous cell cancer in the paraffin blocks. DNA was extracted from $10 \mathrm{~mm}$ sections of paraffin blocks as previously described [23]. The DNA samples were screened for MET Y1253D using real-time polymerase chain reaction (PCR) along with peptide nucleic acid (PNA)based DNA clamping and melting curve analysis as previously described [20]. Briefly, amplified MET wild-type and mutated MET Y1253D were distinguished using melting curve analysis. Addition of the PNA to the reaction mixture allowed detection of the presence of MET Y1253D mutation within a background of at least $98 \%$ wild type plasmid. 154 bp fragment corresponding to MET exon 19, where Y1253D is located, was amplified and analyzed by running a melting curve. The amplification primers were $M E T \times 19$ F: $5^{\prime}$ - ATTCACAGTCAAGGTTGCTGATT and MET $\times$ 19 R: 5' - CTTACCACATCTGACTTGGTGGTA. A pair of hybridization probes were used for the detection of the amplified PCR product. The anchor probe was $3^{\prime}$-terminal blocked against extension (phosphate) and contained a $5^{\prime}$ terminal LightCycler ${ }^{\circledR}$ Red-640 label: LC640-TCATACA TGTCTCTGGCAAGACCA-PH. The sensor probe that was designed to fully match the allele that harbours the T3757G substitution was $3^{\prime}$-terminally labelled with fluorescein: $5^{\prime}$ GTTGTGTACACTATCGTATTCTTT-F (mutated base is underlined). The wild-type-specific PNA oligomer 5'-TA CACTATAGTATTCTTT was present $(9 \mu \mathrm{M})$ to suppress the amplification of the wild-type allele. Real-time PCR was performed with a LightCycler ${ }^{\circledR}$ (Roche Diagnostics) using FastStart DNA reagents (Roche). PCR was performed as follows: 1 cycle for $10 \mathrm{~min}$ at $95^{\circ} \mathrm{C}$ (enzyme activation), 65 cycles with $10 \mathrm{~s}$ at $95^{\circ} \mathrm{C}$ (denaturation) and $40 \mathrm{~s}$ at $57^{\circ} \mathrm{C}$ (annealing of amplification primers and hybridization probes, elongation). Melting analysis was performed by $30 \mathrm{~s}$ heating at $95^{\circ} \mathrm{C}, 150 \mathrm{~s}$ incubation at $40^{\circ} \mathrm{C}$ and slowly heating $\left(0.1^{\circ} \mathrm{C} / \mathrm{s}\right)$ from 40 to $70^{\circ} \mathrm{C}$ and subsequent fluorescence monitoring. Results were processed using the LightCycler $^{\circledR}$ software package (Version 3.5.28).

\section{Statistical analysis}

The primary endpoint was development of distant metastasis. Other endpoints of interest were LRFS and OS. All time-to-event end points were read as the time from the trial registration until either a positive distant metastasis result for DMFS, local tumor progression after incomplete remission and/or local relapse after complete remission for LRFS, or death for OS. For calculation of the median follow-up all patients were used, with the patients who died being censored at the time of death. Patients not reaching an event were censored at the time they were last known to be alive. Continuous baseline variables were dichotomized as follows; age ( $<54$ vs. $\geq 54$ years), $T$-category $\left(\mathrm{T}_{1 / 2 / 3} \mathrm{vs}\right.$. $\mathrm{T}_{4}$ ), N-category $\left(\mathrm{N}_{0 / 1}\right.$ vs. $\mathrm{N}_{2 / 3}$ ) and disease site (oropharynx vs. other). T-categories were grouped to achieve an equal number of events in both sets of patients. The association between all baseline variables and MET Y1253D status were assessed using the Chi-squared or Fisher's exact test. Cox regression analysis was performed to determine if MET Y1253D status is an independent risk factor for either DMFS, LRFS or OS at both the univariate and multivariate level. Inclusion criteria for covariates and confounders to be entered into the multivariate analysis was a $P$-value $<0.1$ in the univariate analysis. The final model contained the primary variables of interest (MET Y1253D status), variables to be considered of high clinical interest regardless of statistical significance and also variables with a statistical significance level of $5 \%$ or less. If chemotherapy administration was not found to be statistically significant in the model as an explanatory variable it was then included as a stratification variable. All analyses were carried out using the Statistical Analysis Systems (SAS) package (SAS Institute, Cary, NC, USA, version 9.1).

\section{Results}

Patient characteristics and $M E T$ genotyping

Patient characteristics are summarized in Table 1. Median patient age was 54 years (range 49-63 years). Median follow-up time was 5.5 years (range $0.1-12.1$ years). The follow-up was less than 3 months in three patients, the follow-up was less than 6 months in 10 patients. Ten (7\%) patients had a tumor of the oral cavity, $78(51 \%)$ of the oropharynx, $40(26 \%)$ of the hypopharynx and $24(16 \%)$ of the larynx. MET Y1253D was found in 21 patients (prevalence of $14 \%$ ), all of whom were male. MET Y1253D status was associated with male gender $(P=0.045)$ and advanced T-category $(P=0.016)$, but not with N-category or age ( $P=0.877$ and 0.130 , respectively). MET Y1253D was not associated with T-category when $\mathrm{T} 1$ and $\mathrm{T} 2$ tumors were compared with $\mathrm{T} 3$ and $\mathrm{T} 4$ tumors (data not shown). MET Y1253D was also not associated with $\mathrm{N}$-category when node negative patients were compared with node positive patients ( $\mathrm{N}_{0}$ vs. $\mathrm{N}_{1 / 2 / 3}$; data not shown). 
Table 1 Patient baseline characteristics

\begin{tabular}{|c|c|c|c|c|}
\hline \multirow[t]{2}{*}{ Parameter } & \multirow[t]{2}{*}{ Total $(\%)$} & \multicolumn{2}{|c|}{$M E T$ Y1253D (\%) } & \multirow[t]{2}{*}{$P$-valu } \\
\hline & & Negative & Positive & \\
\hline Number of patients & $152(100)$ & $131(86)$ & $21(14)$ & \\
\hline \multicolumn{5}{|l|}{ Sex } \\
\hline Male & $130(86)$ & $109(83)$ & $21(100)$ & 0.045 \\
\hline Female & $22(14)$ & $22(17)$ & 0 & \\
\hline \multicolumn{5}{|l|}{ Age (years) } \\
\hline$<54$ & $74(49)$ & $67(51)$ & $7(33)$ & 0.130 \\
\hline$\geq 54$ & $78(51)$ & $64(49)$ & $14(67)$ & \\
\hline \multicolumn{5}{|l|}{ T-category } \\
\hline $\mathrm{T}_{1 / 2 / 3}$ & $94(62)$ & $86(66)$ & $8(38)$ & 0.016 \\
\hline $\mathrm{T}_{4}$ & $58(38)$ & $45(34)$ & $13(62)$ & \\
\hline \multicolumn{5}{|l|}{ N-category } \\
\hline $\mathrm{N}_{0 / 1}$ & $70(46)$ & $60(46)$ & $10(48)$ & 0.877 \\
\hline $\mathrm{N}_{2 / 3}$ & $82(54)$ & $71(54)$ & $11(52)$ & \\
\hline \multicolumn{5}{|l|}{ Disease site } \\
\hline Oropharynx & $78(51)$ & $65(50)$ & $13(62)$ & 0.538 \\
\hline Other & $74(49)$ & $66(50)$ & $8(38)$ & \\
\hline \multicolumn{5}{|l|}{ Chemotherapy } \\
\hline No & $78(51)$ & $71(54)$ & $7(33)$ & 0.334 \\
\hline Yes & 74 (49) & $60(46)$ & $14(67)$ & \\
\hline
\end{tabular}

Tumors were classified according to the tumor-node-metastasis staging system (International Union Against Cancer, edition 4, 1987)

Smoking status, alcohol consumption and tumor differentiation were not available from the study records and thus comparison with MET Y1253D status could not be performed.

\section{MET Y1253D genotype and DMFS}

A total of 29 out of 152 (19\%) HNSCC patients developed distant metastasis after the initial therapy, all occurring within 5 years from trial registration. Distant metastasis were observed at various sites with, by far the most common sites being bone, lung and pleura. Among the 29 patients $28 \%(8 / 29)$ belonged to the MET Y1253D positive group. The 5-year DMFS probability was $72.96 \%$ (95\% confidence interval (CI): 62.68, 80.83). As shown by Kaplan-Meier plot (Fig. 1a), the 5-year DMFS rates were $77.28 \%$ (95\% CI: 66.48, 84.98) for those negative for MET Y1253D, and 44.13\% (95\% CI: 16.08, 69.34) for those positive for MET Y1253D (hazard ratio (HR): 3.016 (95\% CI: 1.332, 6.832); log-rank $P=0.005$ ).

Twenty-six patients underwent salvage surgery ( 3 were MET Y1253D positive, 23 were MET Y1253D negative). Of these, only one MET Y1253D negative patient developed distant metastasis.
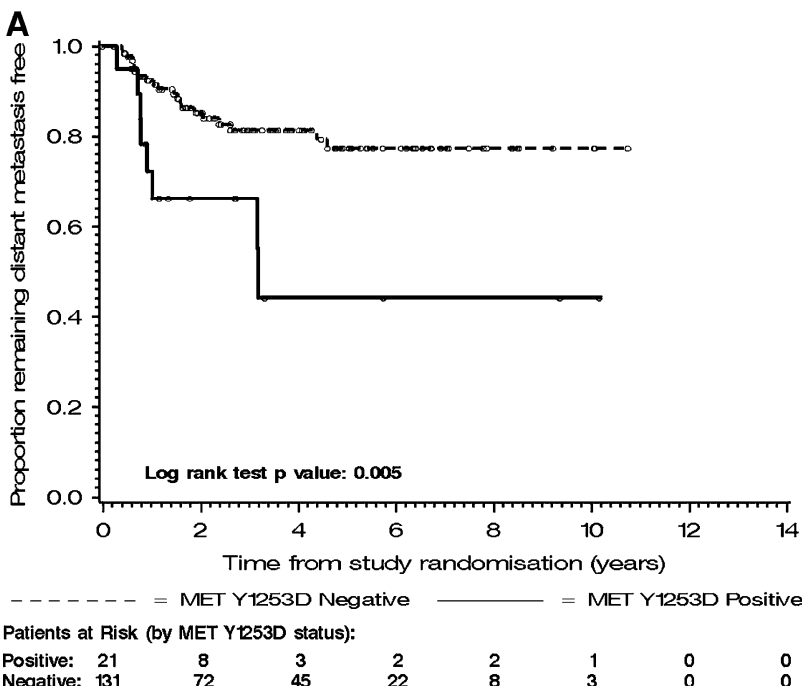

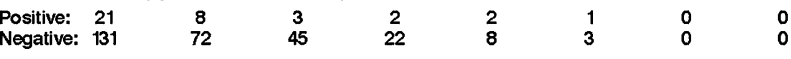
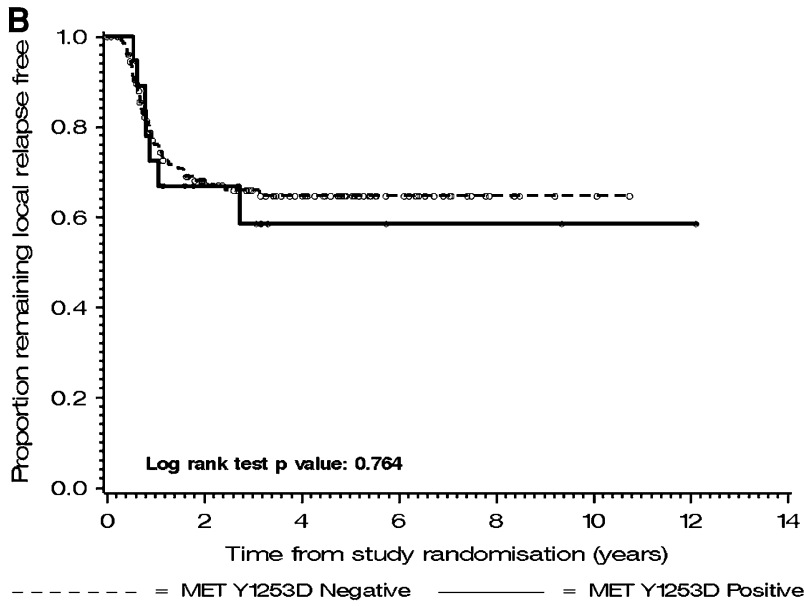
Patients at Risk (by MET Y 253D status):

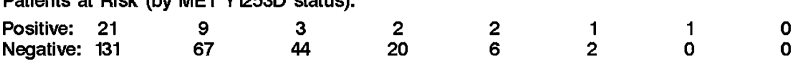

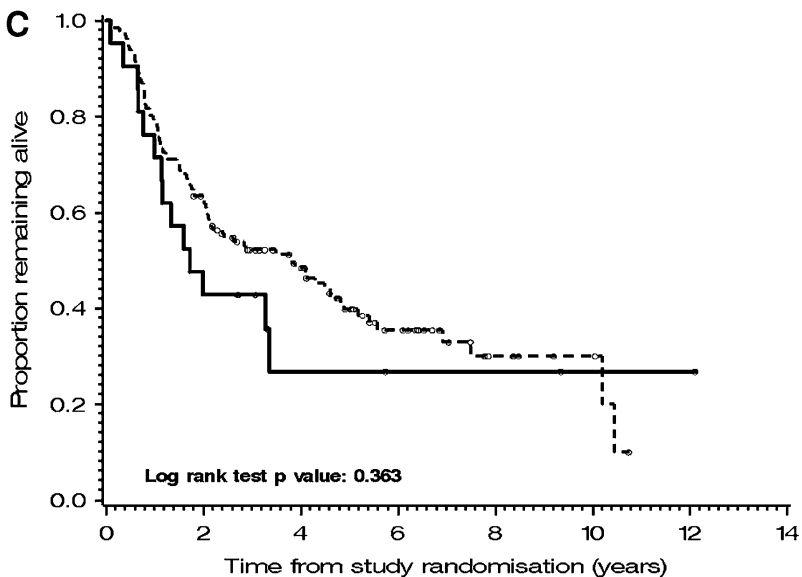

------- = MET Y1253D Negative - = MET Y1253D Positive Patients at Risk (by MET Y 253D status):

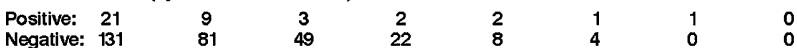

Fig. 1 Actuarial analysis of 5 year a distant metastasis-free survival, b local relapse-free survival and c overall survival with the patients being stratified according to their MET Y1253D status 
In the univariate analysis, advanced T-category was also found to be associated with a statistically significant HR: 2.452 (95\% CI: $1.179,5.101)(P=0.016)$.

In the multivariate analysis using the Cox regression model adjusted for T-category and stratified by chemotherapy administration, MET Y1253D was independently associated with decreased DMFS, HR 2.487 (95\% CI: 1.062, 5.822) $(P=0.036)$ (Table 2$)$.

\section{MET Y1253D genotype and LRFS}

Among the 152 HNSCC patients, 48 (32\%) developed a local relapse after completion of therapy. The KaplanMeier plots showed no significant difference in patients LRFS according to MET Y1253D status, log rank $P=0.764$ (Fig. 1b). Decreased LRFS was associated with female gender (HR: 2.099 (95\% CI: 1.070, 4.119) $(P=0.031)$ ), advanced T-category (HR: 2.040 (95\% CI: 1.157, 3.598) $(P=0.014))$ and absence of chemotherapy administration (HR: $0.406 \quad(95 \%$ CI: $0.218, \quad 0.757)$ $(P=0.005))$ at a univariate level. In the multivariate Cox regression model both the $\mathrm{T}$-category and chemotherapy administration were associated with LRFS (Table 2).

\section{MET Y1253D genotype and OS}

Ninety-five out of $152(62.5 \%)$ HNSCC patients died during the follow-up with a median OS time of 3.35 years (95\% CI: 2.06, 4.59). OS was not significantly different according to the MET Y1253D status, $\log$ rank $P=0.363$ (Fig. 1c) however, OS was associated with advanced T-category (HR: 1.886 (95\% CI: 1.254, 2.838) $(P=$ 0.002)). The Cox regression model in the multivariate analysis produced similar results for the T-category when stratified for chemotherapy administration (Table 2).
At the time of death, of the MET Y1253D negative patients 24 had local relapse only, 11 had distant metastasis only and 9 had both local relapse and distant metastasis. For the MET Y1253D positive patients 3 had local relapse only, 3 had distant metastasis only and 3 had both local relapse and distant metastasis.

\section{Discussion}

Our data indicate that MET Y1235D point mutation is an independent genetic risk factor for the development of distant metastasis after completion of definitive radiation therapy in advanced HNSCC patients. These results have been obtained by correlation of MET Y1235D occurrence and disease outcome within a cohort of patients from a prospective randomized trial of 152 HNSCC patients with a median follow-up of 5.5 years. We had previously described a MET Y1253D prevalence of $10.9 \%$ in a retrospective cohort of 138 HNSCC patients of whom all had tumors of the oropharynx and of whom $77 \%$ were treated by RT alone and $23 \%$ by combined RCT with a median follow-up of the surviving patients of 2.6 years [20]. In this former study there was an association between $M E T$ Y1253D and distant metastasis (HR: 2.41), which, however, did not reach statistical significance. In line with the retrospective results, the current study revealed a similar prevalence of MET Y1253D (14\%) with a comparable hazard ratio (3.016) for DMFS, now reaching statistical significance and strengthening the assumption that the impact of MET Y1253D on the development of distant metastasis in advanced HNSCC is real. While the retrospective study showed a significant association between the presence of MET Y1253D and local failure, the current analysis failed to confirm this finding. The absence of an

Table 2 Treatment outcome analysis following radical radiotherapy \pm chemotherapy of HNSCC

\begin{tabular}{llcr}
\hline Factor & \multicolumn{4}{l}{ Cox regression analysis [hazard ratio $(95 \%$ confidence interval) $(P$-value $)]$} \\
\cline { 2 - 4 } & DMFS* & LRFS & Overall survival* \\
\hline Univariate analysis & & & \\
Sex: female & $0.956(0.333,2.749)(0.934)$ & $2.099(1.070,4.119)(0.031)$ & $0.932(0.508,1.710)(0.820)$ \\
Age (years): $\geq 54$ & $1.057(0.508,2.200)(0.882)$ & $0.604(0.340,1.072)(0.085)$ & $0.847(0.566,1.267)(0.419)$ \\
T-category: $\mathrm{T}_{4}$ & $2.452(1.179,5.101)(0.016)$ & $2.040(1.157,3.598)(0.014)$ & $1.886(1.254,2.838)(0.002)$ \\
N-category: $\mathrm{N}_{2 / 3}$ & $1.624(0.767,3.440)(0.206)$ & $1.000(0.568,1.763)(0.999)$ & $1.171(0.782,1.754)(0.444)$ \\
Chemotherapy: yes & $0.702(0.334,1.474)(0.350)$ & $0.406(0.218,0.757)(0.005)$ & $0.711(0.471,1.074)(0.105)$ \\
$M E T$ Y1253D: positive & $3.016(1.332,6.832)(0.008)$ & $1.130(0.507,2.520)(0.766)$ & $1.303(0.736,2.306)(0.364)$ \\
Multivariate analysis & & & \\
MET Y1253D: positive & $2.487(1.062,5.822)(0.036)$ & $0.894(0.389,2.053)(0.791)$ & $1.203(0.670,2.160)(0.535)$ \\
T-category: $\mathrm{T}_{4}$ & $2.117(0.987,4.539)(0.054)$ & $2.023(1.122,3.647)(0.019)$ & $1.924(1.266,2.925)(0.002)$ \\
Chemo: yes & & $0.416(0.223,0.775)(0.006)$ &
\end{tabular}

* Stratified by chemotherapy administration in the multivariate analysis 
association between LRFS and MET Y1253D status may be caused by differences in the study populations i.e., treatment site, treatment and follow-up (in the context of comparison of a retrospective and prospective trial). To adjust for some of these imbalances we performed a subgroup analysis in oropharynx patients (78/152) but again there was no association between LRFS and MET Y1253D found (data not shown). Since the median followup of the retrospective study was 2.6 years, we calculated for the current study also the 3-year actuarial DMFS, LRFS and OS rates which, however, revealed comparable results to the 5-year data.

The fact that there was no female patient with a $M E T$ Y1253D point mutation could possibly be attributed to a different mechanism of cancer development as it has been stated by us and others $[23,24]$. One limitation of our study is the fact that the MET Y1253D mutation was only found in $14 \%$ of the patients and distant metastasis occurred only in $19 \%$. This limits the statistical significance of our results. Another limitation is the lack of prospective stratification according to the MET Y1253D status within the prospective study, thus the incidence of the mutation in the treatment group was different (Table 1). Further, metastatic tissue was not available for analysis, thus the MET Y1253D status was only assessed in primary tumors. It would be highly desirable indeed to analyse metastases for the presence of mutations; however, there is a very high barrier from ethical committees to submit patients for invasive procedures such as removal of tumor tissue for scientific reasons without direct clinical implications. However, it has been shown by others that the incidence of MET Y1253D is higher in metastatic tissue as compared to the corresponding primary tumors which suggests that cells expressing mutant MET undergo clonal expansion during HNSCC progression [19].

Despite significant improvement in local tumor control, distant metastasis-free survival and overall survival in head and neck cancers during the last years, the development of distant metastasis is still a major clinical problem and the development of new therapeutic strategies is therefore of great interest. Previous studies have associated both MET receptor expression as well as somatic mutations of the $M E T$ oncogene with invasive growth and distant metastasis [13-15]. MET signaling can be inhibited by various approaches, including inhibition of ligand-receptor interaction, inhibition of tyrosine kinase catalytic activity and inhibition of receptor-effector interactions [16, 18, 25, 26]. Various MET inhibitors are currently undergoing development in phase I to II studies in cancer. The association between MET Y1253D status and DMFS strongly points towards a potential role for MET inhibitors as a novel specific treatment strategy to reduce the risk of distant metastasis in patients with advanced head and neck cancers.
Acknowledgments This study was supported by a grant from Oncosuisse (OCS—01681-02-2005) to DMA and YZ.

\section{References}

1. Pignon JP, Bourhis J, Domenge C et al (2000) Chemotherapy added to locoregional treatment for head and neck squamous-cell carcinoma: three meta-analyses of updated individual data. MACH-NC collaborative group. Meta-analysis of chemotherapy on head and neck cancer. Lancet 355:949-955

2. Denis F, Garaud P, Bardet E et al (2004) Final results of the 9401 French head and neck oncology and radiotherapy group randomized trial comparing radiotherapy alone with concomitant radiochemotherapy in advanced-stage oropharynx carcinoma. J Clin Oncol 22:69-76

3. Forastiere AA, Goepfert H, Maor M et al (2003) Concurrent chemotherapy and radiotherapy for organ preservation in advanced laryngeal cancer. N Engl J Med 349:2091-2098

4. Paccagnella A, Orlando A, Marchiori C et al (1994) Phase III trial of initial chemotherapy in stage III or IV head and neck cancers: a study by the Gruppo di Studio sui Tumori della Testa e del Collo. J Natl Cancer Inst 86:265-272

5. Zorat PL, Paccagnella A, Cavaniglia G et al (2004) Randomized phase III trial of neoadjuvant chemotherapy in head and neck cancer: 10-year follow-up. J Natl Cancer Inst 96:1714-1717

6. Al-Sarraf M, LeBlanc M, Giri PG et al (1998) Chemoradiotherapy versus radiotherapy in patients with advanced nasopharyngeal cancer: phase III randomized Intergroup study 0099. J Clin Oncol 16:1310-1317

7. Calais G, Alfonsi M, Bardet E et al (1999) Randomized trial of radiation therapy versus concomitant chemoradiotherapy and radiation therapy for advanced-stage oropharynx carcinoma. $\mathrm{J}$ Natl Cancer Inst 91:2081-2086

8. Jeremic B, Shibamoto Y, Milicic B et al (2000) Hyperfractionated radiation therapy with or without concurrent low-dose daily cisplatin in locally advanced squamous cell carcinoma of the head and neck: a prospective randomized trial. J Clin Oncol 18:1458-1464

9. Cohen EE (2006) Role of epidermal growth factor receptor pathway-targeted therapy in patients with recurrent and/or metastatic squamous cell carcinoma of the head and neck. J Clin Oncol 24:2659-2665

10. Egloff AM, Grandis JR (2008) Targeting epidermal growth factor receptor and SRC pathways in head and neck cancer. Semin Oncol 35:286-287

11. Le Tourneau C, Siu LL (2008) Molecular-targeted therapies in the treatment of squamous cell carcinomas of the head and neck. Curr Opin Oncol 20:256-263

12. Baselga J, Trigo JM, Bourhis J et al (2005) Phase II multicenter study of antiepidermal growth factor receptor monoclonal antibody cetuximab in combination with platinum-based chemotherapy in patients with platinum-refractory metastatic and/or recurrent squamous cell carcinoma of the head and neck. J Clin Oncol 23:5568-5577

13. Danilkovitch-Miagkova A, Zbar B (2002) Dysregulation of Met receptor tyrosine kinase activity in invasive tumors. J Clin Invest 109:863-867

14. Birchmeier C, Birchmeier W, Gherardi E et al (2003) Met, metastasis, motility and more. Nat Rev Mol Cell Biol 4:915-925

15. Benvenuti S, Comoglio PM (2007) The MET receptor tyrosine kinase in invasion and metastasis. J Cell Physiol 213:316-325

16. Corso S, Migliore C, Ghiso E et al (2008) Silencing the MET oncogene leads to regression of experimental tumors and metastases. Oncogene 27:684-693 
17. Jiang WG, Martin TA, Parr C et al (2005) Hepatocyte growth factor, its receptor, and their potential value in cancer therapies. Crit Rev Oncol Hematol 53:35-39

18. Comoglio PM, Giordano S, Trusolino L (2008) Drug development of MET inhibitors: targeting oncogene addiction and expedience. Nat Rev Drug Discov 7:504-506

19. Di Renzo MF, Olivero M, Martone T (2000) Somatic mutations of the MET oncogene are selected during metastatic spread of human HNSC carcinomas. Oncogene 19:1547-1555

20. Aebersold DM, Landt O, Berthou S et al (2003) Prevalence and clinical impact of Met Y1253D-activating point mutation in radiotherapy-treated squamous cell cancer of the oropharynx. Oncogene 22:8519-8523

21. Lorenzato A, Olivero $M$, Patanè $S$ et al (2002) Novel somatic mutations of the MET oncogene in human carcinoma metastases activating cell motility and invasion. Cancer Res 62:7025
22. Huguenin P, Beer KT, Allal A et al (2004) Concomitant cisplatin significantly improves locoregional control in advanced head and neck cancers treated with hyperfractionated radiotherapy. J Clin Oncol 22:4665-4673

23. Lindel K, Beer KT, Laissue J et al (2001) Human papillomavirus positive squamous cell carcinoma of the oropharynx: a radiosensitive subgroup of head and neck carcinoma. Cancer 92:805813

24. Braakhuis BJ, Snijders PJ et al (2004) Genetic patterns in head and neck cancers that contain or lack transcriptionally active human papillomavirus. J Natl Cancer Inst 96:998-1006

25. Cassinelli G, Lanzi C, Petrangolini G et al (2006) Inhibition of cMet and prevention of spontaneous metastatic spreading by the 2indolinone RPI-1. Mol Cancer Ther 5:2388-2397

26. Peruzzi B, Bottaro DP (2006) Targeting the c-Met signaling pathway in cancer. Clin Cancer Res 12:3657 